\title{
GOLOMB'S ARITHMETICAL SEMIGROUP TOPOLOGY AND A SEMIPRIME SUFFICIENCY CONDITION FOR DIRICHLET'S THEOREM
}

\author{
CHRIS ORUM
}

\begin{abstract}
Dirichlet's theorem on the distribution of primes in arithmetic progressions states that every positive integer sequence $\{a n+b \mid n \geq 0\}$ with $a$ and $b$ coprime contains infinitely many primes. In 1959, Golomb pointed out that, by taking such arithmetic progressions as a base for a topology $\mathcal{D}$ on the positive integers, the resulting topological space $\left(\mathbb{Z}^{+}, \mathcal{D}\right)$ is both Hausdorff and connected. More recently, Knopfmacher and Porubsky showed that $\left(\mathbb{Z}^{+}\right.$, $\mathcal{D})$ is a topological semigroup under multiplication. After revisiting this result, we show that Dirichlet's theorem is implied by the statement that the $\mathcal{D}$-closure of the primes contains the semiprimes.
\end{abstract}

1. Introduction. In [4], Golomb demonstrated a topological approach to Dirichlet's theorem on the distribution of primes in arithmetic progressions, working with a space previously considered by Brown [2]. A topology $\mathcal{D}$ on the positive integers $\mathbb{Z}^{+}$is defined, by taking as a base for $\mathcal{D}$, those sets of the form $\{a n+b\}$ with $(a, b)=1$. Then, Dirichlet's theorem is equivalent to the statement that the primes $P$ are dense in $\left(\mathbb{Z}^{+}, \mathcal{D}\right)[\mathbf{4}$, Theorem 6].

The space $\left(\mathbb{Z}^{+}, \mathcal{D}\right)$ is Hausdorff $[\mathbf{4}$, Theorem 2] and connected [2], [4, Theorem 3], but not locally connected [6, Theorem 1]. On the other hand, the related space $\left(\mathbb{Z}^{+}, \mathcal{D}^{\prime}\right)$ subsequently introduced by Kirch [6] is Hausdorff, connected and locally connected. A base for $\mathcal{D}^{\prime}$ consists of arithmetic progressions $\{a n+b\}$ such that $(a, b)=1$ and $a$ is square-free [6, Corollary to Theorem 4]. These spaces appear in the collection of counterexamples given by Steen and Seebach [10] with

2010 AMS Mathematics subject classification. Primary 11A41, 11B25, 11N13, $54 \mathrm{H} 99$.

Keywords and phrases. Dirichlet's theorem, arithemetic progressions, primes, topological semigroup.

Received by the editors on December 9, 2013, and in revised form on July 29, 2014.

DOI: $10.1216 / \mathrm{RMJ}-2016-46-3-939$

Copyright (C)2016 Rocky Mountain Mathematics Consortium 
the names Relatively Prime Integer Topology $(\mathcal{D})$ and Prime Integer Topology $\left(\mathcal{D}^{\prime}\right)$. Further analysis of these and related spaces is given in $[1,7,8,11,12,13]$.

The purpose of this paper is twofold. First, we revisit a result in [7] that deserves to be better known: that the topology $\mathcal{D}$ endows $\mathbb{Z}^{+}$with the structure of a topological semigroup. Second, we use the topological semigroup structure to prove the following sufficiency condition for the density of $P$ involving the semiprimes, $P_{2}=\{4,6,9, \ldots\}$. These are the positive integers with exactly two, not necessarily distinct, prime factors. More generally, we write $P_{k}, k \geq 2$, for the set of $k$-almost primes. These are the positive integers with exactly $k$ prime factors.

Theorem 1.1. If $P_{2} \subseteq \bar{P}$, then $\bar{P}=\mathbb{Z}^{+}$.

In other words, in order to prove that the set $P$ of prime numbers is dense in $\left(\mathbb{Z}^{+}, \mathcal{D}\right)$, it is sufficient to prove that the closure of $P$ contains the semiprimes.

Corollary 1.2. Let $a$ and $b$ denote positive integers. The following statements are equivalent:

(i) Every arithmetic progression of the form $\{a n+b \mid n \geq 0\}$, with $(a, b)=1$, that contains at least one semiprime, also contains a prime.

(ii) Every arithmetic progression of the form $\{a n+b \mid n \geq 0\}$, with $(a, b)=1$, contains infinitely many primes.

Theorem 1.1 is a consequence of the fact that $\left(\mathbb{Z}^{+}, \times, \mathcal{D}\right)$ is a topological semigroup. This fact is established in [7] by Knopfmacher and Porubsky who work with coset topologies defined on a given integral domain $R$ (or a more general type of ring) and related topologies on the set $R^{0}=R \backslash\{0\}$ and the set $R^{0} / \sim$ of its associate classes. Here we reprove continuity of multiplication on $\left(\mathbb{Z}^{+}, \mathcal{D}\right)$ by a somewhat different approach that involves lifting continuity from multiplication on the quotient rings of $\mathbb{Z}$. This is given as the proof of Lemma 2.6. Theorem 1.1 then follows using the fact that $\left(\mathbb{Z}^{+}, \mathcal{D}\right)$ is Hausdorff and connected, and general properties of topological semigroups that are not specific to the arithmetical properties of $\left(\mathbb{Z}^{+}, \mathcal{D}\right)$. 
Unfortunately, it appears unlikely that Theorem 1.1 can be used to give an independent proof of Dirichlet's theorem. The approach of using novel topologies on the positive integers is still lacking in "powerful new ideas and methods" as put forward by Golomb over 50 years ago (cf., [4]).

\section{Main results.}

2.1. Notation and preliminaries. We regard the positive integers $\mathbb{Z}^{+}$as a multiplicative subsemigroup of $\left(\mathbb{Z}^{0}, \times\right)$, where $\mathbb{Z}^{0}=\mathbb{Z} \backslash\{0\}$ denotes the nonzero integers. Arithmetic progressions of the form $\{a n+b \mid n \geq 0\}$ with $a, b \in \mathbb{Z}^{+}$are denoted by $U_{a}(b)$. This notation implies $a, b>0$. A base for Golomb's topology on $\mathbb{Z}^{+}$is the collection $\mathcal{B}=\left\{U_{a}(b) \mid(a, b)=1\right\}$. Here, $(a, b)$ is the greatest common denominator of $a$ and $b$. Assuming $b<a$ has no effect on the resulting topology:

\section{Proposition 2.1. Let}

$$
\Sigma_{1}=\left\{U_{a}(b) \mid(a, b)=1, b<a\right\} .
$$

Then, $\mathcal{B}$ and $\Sigma_{1}$ generate the same topology.

Proof. Let $\mathcal{D}_{1}$ be the topology generated by $\Sigma_{1}$. Since $\Sigma_{1} \subseteq \mathcal{B}$, it follows that $\mathcal{D}_{1} \subseteq \mathcal{D}$. If $(a, b)=1$ and $b>a$, let $\left\{q_{k}\right\}_{k=0}^{\infty}$ be a sequence of primes chosen to satisfy $q_{k}>a k+b$ for all $k \geq 0$. Then, since $\left(q_{k}, a k+b\right)=1$ for all $k \geq 0$, and $(a, a k+b)=1$ for all $k \geq 0$, it follows that $\left(a q_{k}, a k+b\right)=1$ for all $k \geq 0$. We also have $a q_{k} \geq q_{k}>a k+b$. Consequently,

$$
U_{a}(b)=\bigcup_{k=0}^{\infty} U_{a q_{k}}(a k+b)
$$

is a representation of $U_{a}(b) \in \mathcal{B}$ as a union over members of $\Sigma_{1}$. This shows that $\mathcal{D} \subseteq \mathcal{D}_{1}$. Hence, $\mathcal{D}=\mathcal{D}_{1}$.

We denote by $h_{N}: \mathbb{Z} \rightarrow \mathbb{Z} / N \mathbb{Z}$, the ring homomorphism that sends $x \in \mathbb{Z}$ to its residue class,

$$
h_{N}(x)=\{x+m N \mid m \in \mathbb{Z}\} .
$$




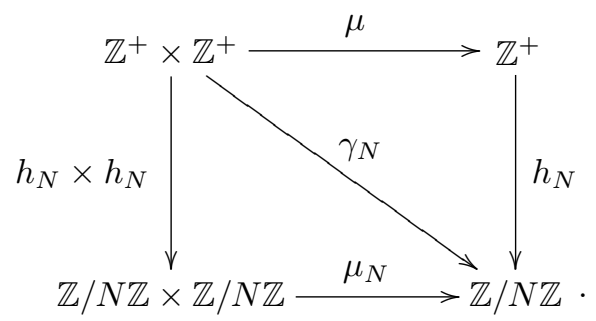

FIGURE 1. Lifting continuity of multiplication from the quotient rings.

We index the collection of all such homomorphisms by $N \geq 1$. We write $\bar{x}$ for $h_{N}(x)$ in contexts where $N$ is unambiguous. We let $\mu: \mathbb{Z} \times \mathbb{Z} \rightarrow \mathbb{Z}$ denote multiplication and we let

$$
\mu_{N}: \mathbb{Z} / N \mathbb{Z} \times \mathbb{Z} / N \mathbb{Z} \longrightarrow \mathbb{Z} / N \mathbb{Z},
$$

denote multiplication on the quotient ring. We also write $h_{N}$ for the restriction $\left.h_{N}\right|_{\mathbb{Z}^{+}}$, and $\mu$ for $\left.\mu\right|_{\mathbb{Z}^{+} \times \mathbb{Z}^{+}}$. Then, for all $N \geq 1$, the diagram in Figure 1 is commutative. In particular,

$$
\gamma_{N}=\mu_{N} \circ\left(h_{N} \times h_{N}\right)=h_{N} \circ \mu .
$$

We equip each quotient $\operatorname{ring} \mathbb{Z} / N \mathbb{Z}, N \geq 1$, with the invertible element topology $\mathcal{E}_{N}$. A base for $\mathcal{E}_{N}$ is $\mathbb{Z} / N \mathbb{Z}$, along with all sets of the form $\{\bar{u}\}$, where $\bar{u} \in U(\mathbb{Z} / N \mathbb{Z})$, i.e., $\bar{u}$ belongs to the group of units of the quotient ring. Equivalently,

$$
\emptyset \neq A \subseteq \mathbb{Z} / N \mathbb{Z}
$$

is closed if and only if $A$ contains all zero divisors in $\mathbb{Z} / N \mathbb{Z}$. Next, recalling the notion of an initial topology, we let $\mathcal{D}_{w}$ be the weakest topology on $\mathbb{Z}^{+}$that makes each $h_{N}$ continuous. We will show that $\mathcal{D}_{w}=\mathcal{D}$.

Definition 2.2. Let

$$
\left\{f_{i}: X \longrightarrow\left(Y_{i}, \mathcal{Y}_{i}\right) \mid i \in I\right\}
$$

be a family of functions where $X$ is any set, $\left(Y_{i}, \mathcal{Y}_{i}\right)$ are topological spaces and $I$ is an index set. The smallest topology $\mathcal{T}$ on $X$ that makes each $f_{i}$ continuous is called the initial topology. 
Proposition 2.3. Golomb's topology $\mathcal{D}$ coincides with the initial topology $\mathcal{D}_{w}$ generated by the family of maps

$$
h_{N}: \mathbb{Z}^{+} \longrightarrow\left(\mathbb{Z} / N \mathbb{Z}, \mathcal{E}_{N}\right), \quad N \geq 1 .
$$

Proof. Any collection of sets of the form $h_{N}^{-1}(S)$, where $S$ runs over a subbase of $\mathcal{E}_{N}$ and $N$ runs over the index set, will generate the initial topology $\mathcal{D}_{w}$, e.g., $[\mathbf{9}$, page 79$]$. By letting $S$ run over the sets $\{\bar{u}\}$,

$$
\bar{u} \in U(\mathbb{Z} / N \mathbb{Z}), \quad N \geq 1,
$$

we obtain the collection $\Sigma_{1}$ defined in Proposition 2.1, which generates $\mathcal{D}$. It follows that $\mathcal{D}_{w}=\mathcal{D}$.

2.2. The topological semigroup and proofs. Our approach to Theorem 1.1 draws on the following property of initial topologies. This property actually characterizes them, as is well known, see e.g., [9, page 138].

Theorem 2.4. [5, page 126]. Let $X$ have the initial topology determined by a family of functions

$$
\left\{f_{i}: X \longrightarrow Y_{i} \mid i \in I\right\},
$$

where each $Y_{i}$ is a topological space and $I$ is an index set. Then, for any space $Z$, a function $g: Z \rightarrow X$ is continuous if and only if, for each $i \in I$, the composition $f_{i} \circ g: Z \rightarrow Y_{i}$ is continuous.

Definition 2.5. A topological semigroup $(S, \circledast, \mathcal{T})$ is a semigroup $S=(S, \circledast)$, provided with a Hausdorff topology $\mathcal{T}$, such that the semigroup operation $(x, y) \rightarrow x \circledast y$ is continuous with the product topology on $S \times S$.

The operation $x \circledast y$ is often written as $x y$. We follow [3] in stipulating that the topology be Hausdorff, but not all authors require this. To verify continuity of multiplication it is sufficient to show that, for each $x, y \in S$ and each open set $W \subseteq S$, such that $x y \in W$, there exist open sets $U=U_{x}$ and $V=V_{y}$ such that $x \in U, y \in V$ and

$$
U V=\{u v \mid u \in U, v \in V\} \subseteq W .
$$


Indeed, if this holds, then the preimage of an open set $W \subseteq S$, under the inverse of the $\circledast$-operation, is the open set

$$
\bigcup\left\{U_{x} \times V_{y} \mid x y \in W\right\} .
$$

Lemma 2.6. With multiplication $\times$ as the semigroup operation, $\left(\mathbb{Z}^{+}, \times, \mathcal{D}\right)$ is a topological semigroup.

Proof. We equip each $\mathbb{Z} / N \mathbb{Z}$ with the topology $\mathcal{E}_{N}$ and

$$
\mathbb{Z} / N \mathbb{Z} \times \mathbb{Z} / N \mathbb{Z}
$$

with the corresponding product topology. An element $\overline{x y} \in \mathbb{Z} / N \mathbb{Z}$ is either a unit or zero divisor. It is a unit if and only if $\bar{x}$ and $\bar{y}$ are both units, in which case, we take $U=\{\bar{x}\}$ and $V=\{\bar{y}\}$. If $\overline{x y}$ is a zero divisor, then the only open neighborhood of $\overline{x y}$ is $\mathbb{Z} / N \mathbb{Z}$, and we take

$$
U=V=\mathbb{Z} / N \mathbb{Z} \text {. }
$$

Thus, $\mu_{N}$ is continuous. With the topology $\mathcal{D}$ on $\mathbb{Z}^{+}$and the product topology on $\mathbb{Z}^{+} \times \mathbb{Z}^{+}$, the maps $h_{N}$ and $h_{N} \times h_{N}$ in Figure 1 are continuous. Then,

$$
\gamma_{N}=\mu_{N} \circ\left(h_{N} \times h_{N}\right)
$$

is continuous for each $N \geq 1$, and the continuity of each $\gamma_{N}=h_{N} \circ \mu$, along with Theorem 2.4, implies the continuity of

$$
\mu: \mathbb{Z}^{+} \times \mathbb{Z}^{+} \longrightarrow \mathbb{Z}^{+} .
$$

Proof of Theorem 1.1. Let

$$
M=\left\{x \in \mathbb{Z}^{+} \mid x P \subseteq \bar{P}\right\} .
$$

First, we show that $M$ is closed. If

$$
M^{c}:=\mathbb{Z}^{+} \backslash M=\emptyset,
$$

then $M$ is closed. If $M^{c} \neq \emptyset$, let $a \in M^{c}$. Then, for some $p \in P$, we have $a p \notin \bar{P}$. Continuity of multiplication guarantees the existence of open sets $U$ and $V$ such that $a \in U, p \in V$, and $U V \subseteq(\bar{P})^{c}$. In particular,

$$
U p=\{z p \mid z \in U\} \subseteq(\bar{P})^{c} .
$$


Accordingly, $U \subseteq M^{c}$ (by definition of $M$ ) and $U$ is an open neighborhood of $a \in M^{c}$, which implies that $M^{c}$ is open. So again, $M$ is closed.

We now apply the hypothesis $P_{2} \subseteq \bar{P}$. This implies that $P \subseteq M$. Since $\bar{P}$ is the smallest closed set containing $P$, it follows that $\bar{P} \subseteq M$. Thus, for any $x \in \bar{P}$ and $p \in P$, we know that $x p \in \bar{P}$. In particular, letting $k \geq 1$ be fixed but arbitrary and considering

$$
P_{k} P=\left\{x p \mid x \in P_{k}, p \in P\right\}=P_{k+1},
$$

we obtain the implication:

$$
P_{k} \subseteq \bar{P} \Longrightarrow P_{k+1} \subseteq \bar{P} .
$$

This implication holds for all $k \geq 1$. We now proceed by induction. Taking $P_{1}=P \subseteq \bar{P}$ as the base step and equation (2.1) as the induction step yields:

$$
\mathbb{Z}^{+} \backslash\{1\}=\bigcup_{k=1}^{\infty} P_{k} \subseteq \bar{P} .
$$

We rule out $\bar{P}=\mathbb{Z}^{+} \backslash\{1\}$; otherwise, $\mathbb{Z}^{+}=\bar{P} \cup\{1\}$ would be the union of two disjoint closed sets, violating the connectedness of $\mathbb{Z}^{+}$ (which, we recall, was shown in [4, Theorem 3]; see also [11, Theorem 3.3]). Hence, $\bar{P}=\mathbb{Z}^{+}$.

Remark 2.7. Elementary proofs of the fact that $U_{m}(1) \cap P \neq \emptyset$ for all $m \geq 1$ are known (e.g., [14, Corollary 2.11]). Using this, we observe, in order to conclude that $\bar{P}=\mathbb{Z}^{+}$, it is sufficient to show that $P_{k+1} \subseteq \bar{P}$ for some $k \geq 1$. Indeed, the set

$$
M_{k}=\left\{x \in \mathbb{Z}^{+} \mid x P_{k} \subseteq \bar{P}\right\},
$$

may be shown to be closed by arguing as in the preceding proof. Then $P_{k+1} \subseteq \bar{P}$ implies $P \subseteq M_{k}$, and hence, $\bar{P} \subseteq M_{k}$. Moreover,

$$
\bar{P} P_{k}=\left\{p q_{k} \mid p \in \bar{P}, q_{k} \in P_{k}\right\} \subseteq \bar{P},
$$

which implies that $P_{k} \subseteq \bar{P}$ using $1 \in \bar{P}$. Iterating this argument establishes that $P_{2} \subseteq \bar{P}$, and then $\bar{P}=\mathbb{Z}^{+}$by Theorem 1.1. 
3. Extensions. The proof of continuity of multiplication on $\left(\mathbb{Z}^{+}\right.$, $\mathcal{D})$ readily extends to the class of spaces of the form $\left(\mathbb{Z}^{+}, \mathcal{X}\right)$ that arises in the following way:

(i) each quotient ring $\mathbb{Z} / N \mathbb{Z}$ is equipped with a topology $\mathcal{X}_{N}$ such that multiplication is continuous on $\left(\mathbb{Z} / N \mathbb{Z}, \mathcal{X}_{N}\right)$,

(ii) the topology $\mathcal{X}$ on $\mathbb{Z}^{+}$is the initial topology generated by the family of maps

$$
h_{N}: \mathbb{Z}^{+} \longrightarrow\left(\mathbb{Z} / N \mathbb{Z}, \mathcal{X}_{N}\right), \quad N \geq 1 .
$$

The space $\left(\mathbb{Z}^{+}, \mathcal{D}^{\prime}\right)$ is included in this class. So are some of the arithmetical coset topologies discussed in [7], at least if the underlying integral domain is $\mathbb{Z}$.

In this last section, we consider Kirch's topology $\mathcal{D}^{\prime}$ and the arithmetical coset topology $\mathcal{D}^{*}$ (defined below) in this framework.

3.1. Kirch's topology $\mathcal{D}^{\prime}$. A base for Kirch's topology $\mathcal{D}^{\prime}$ on $\mathbb{Z}^{+}$is

$$
\mathcal{B}^{\prime}=\left\{U_{a}(b) \mid(a, b)=1, a \text { is square-free }\right\} .
$$

We will show that $\mathcal{D}^{\prime}$ coincides with an initial topology $\mathcal{D}_{w}^{\prime}$ generated by a family of maps,

$$
h_{N}: \mathbb{Z}^{+} \longrightarrow\left(\mathbb{Z} / N \mathbb{Z}, \mathcal{X}_{N}\right), \quad N \geq 1,
$$

and that the topologies $\mathcal{X}_{N}$ on the quotient rings fulfill requirement (i). Consequently, $\left(\mathbb{Z}^{+}, \times, \mathcal{D}^{\prime}\right)$ is a topological semigroup.

The proof of the following proposition is similar to the proof of Proposition 2.1 and is omitted.

Proposition 3.1. Let

$$
\Sigma_{1}^{\prime}=\left\{U_{a}(b) \mid(a, b)=1, a \text { is square-free, } b<a\right\} .
$$

Then, $\mathcal{B}^{\prime}$ and $\Sigma_{1}^{\prime}$ generate the same topology.

Proposition 3.2. Kirch's topology $\mathcal{D}^{\prime}$ coincides with the initial topology $\mathcal{D}_{w}^{\prime}$, generated by the family of maps

$$
h_{N}: \mathbb{Z}^{+} \longrightarrow\left(\mathbb{Z} / N \mathbb{Z}, \mathcal{X}_{N}\right), \quad N \geq 1,
$$

where $\mathcal{X}_{N}=\mathcal{E}_{N}$ if $N$ is square-free, and trivial otherwise, i.e., $\mathcal{X}_{N}=$ $\{\emptyset, \mathbb{Z} / N \mathbb{Z}\}$ if $N$ is not square-free. 
Proof. As in the proof of Proposition 2.3, the initial topology $\mathcal{D}_{w}^{\prime}$ is generated by sets of the form $h_{N}^{-1}(S)$, where $N$ runs over the index set, and for each fixed $N, S$ runs over a subbase of $\mathcal{X}_{N}$. Accordingly, we let $S$ run over sets of the form $\{\bar{u}\}$,

$$
\bar{u} \in U(\mathbb{Z} / N \mathbb{Z}),
$$

if $N$ is square-free, and let $S=\mathbb{Z} / N \mathbb{Z}$ if $N$ is not square-free. In the latter case, $h_{N}^{-1}(\mathbb{Z} / N \mathbb{Z})$ contributes nothing to the definition of $\mathcal{D}_{w}^{\prime}$; hence, $\mathcal{D}_{w}^{\prime}$ is generated by the collection of sets

$$
\left\{h_{N}^{-1}(\bar{u}) \mid N \geq 1 \text { is square-free, } \bar{u} \in U(\mathbb{Z} / N \mathbb{Z})\right\} .
$$

This is precisely the collection $\Sigma_{1}^{\prime}$ which generates $\mathcal{D}^{\prime}$. Hence, $\mathcal{D}_{w}^{\prime}=$ $\mathcal{D}^{\prime}$.

To complete the argument that $\left(\mathbb{Z}^{+}, \times, \mathcal{D}^{\prime}\right)$ is a topological semigroup, we note that multiplication is continuous on each of the spaces $\left(\mathbb{Z} / N \mathbb{Z}, \mathcal{X}_{N}\right)$. If $N$ is not square-free, then $\mathcal{X}_{N}=\{\emptyset, \mathbb{Z} / N \mathbb{Z}\}$, and checking the continuity of multiplication is trivial. If $N$ is square-free, then $\mathcal{X}_{N}=\mathcal{E}_{N}$, and continuity of multiplication is established in the proof of Lemma 2.6.

3.2. The arithmetical coset topology $\mathcal{D}^{*}$. Our second example is $\left(\mathbb{Z}^{+}, \mathcal{D}^{*}\right)$, which arises as follows: the authors of [7] define, more generally, the invertible coset topology $\tau_{3}$ on a commutative $\operatorname{ring} R$ with identity. A base for $\tau_{3}$ consists of cosets of the form $a+A$, where (a) and $A$ are coprime ideals. Ideals $A$ and $B$ in such a ring are coprime if and only if $a+b=1$ for some $a \in A$ and $b \in B$. If $R$ is an integral domain, then $\tau_{3}$ induces a subspace topology $\tau^{*}$ on $R^{0}:=R \backslash\{0\}$ which converts the multiplicative semigroup $R^{0}$ into a topological semigroup. Moreover, $\tau^{*}$ induces a quotient topology $\Delta^{*}$ on the set $G_{R}=R^{0} / \sim$ of equivalence classes of associates in $R^{0}(a \sim b$ if and only if the ideals $(a)$ and $(b)$ coincide). Then $\left(G_{R}, \Delta^{*}\right)$ is a topological semigroup when $G_{R}$ is equipped with the natural multiplication.

While in general there is no canonical embedding of the semigroup $G_{R}$ into $R^{0}$, if $R=\mathbb{Z}$, then $G_{\mathbb{Z}}$ may be identified with the multiplicative subsemigroup $\mathbb{Z}^{+} \subseteq \mathbb{Z}^{0}:=\mathbb{Z} \backslash\{0\}$. Following [7], we then obtain two topologies on $\mathbb{Z}^{+}$. The first is the subspace topology induced by $\tau^{*}$ because $\mathbb{Z}^{+} \subseteq \mathbb{Z}^{0}$. This coincides with $\mathcal{D}$. The second is the weaker 
(but still Hausdorff) topology $\mathcal{D}^{*} \subsetneq \mathcal{D}$ induced by the quotient topology $\Delta^{*}$ on $G_{\mathbb{Z}}$ and the identification of $G_{\mathbb{Z}}$ with $\mathbb{Z}^{+}$.

We now observe that $\mathcal{D}^{*}$ coincides with the initial topology induced by the maps

$$
h_{N}: \mathbb{Z}^{+} \longrightarrow\left(\mathbb{Z} / N \mathbb{Z}, \mathcal{E}_{N}^{*}\right), \quad N \geq 1 .
$$

A base for $\mathcal{E}_{N}^{*}$ consists of $\mathbb{Z} / N \mathbb{Z}$ and the sets of the form $\{-\bar{u}, \bar{u}\}$ where $\bar{u} \in \mathbb{Z} / N \mathbb{Z}$ is a unit. It is straightforward to check that multiplication is continuous on $\left(\mathbb{Z} / N \mathbb{Z}, \mathcal{E}_{N}^{*}\right)$ for each $N \geq 1$; hence, $\left(\mathbb{Z}^{+}, \times, \mathcal{D}^{*}\right)$ is a topological semigroup (cf., [7, pages 135-136]).

\section{REFERENCES}

1. Kevin A. Broughan, Adic topologies for the rational integers, Canad. J. Math. 55 (2003), 711-723.

2. Morton Brown, A countable connected Hausdorff space, in The April meeting in New York, L.W. Cohen, ed., Bull. Amer. Math. Soc. 59 (1953), 367.

3. James Harvey Carruth, John A. Hildebrant and R.J. Koch, The theory of topological semigroups, Mono. Text. Pure Appl. Math. 75, Marcel Dekker, Inc., New York, 1983.

4. Solomon W. Golomb, A connected topology for the integers, Amer. Math. Month. 66 (1959), 663-665.

5. K.D. Joshi, Introduction to general topology. John Wiley \& Sons, Inc., New York, 1983.

6. A.M. Kirch, A countable, connected, locally connected Hausdorff space, Amer. Math. Month. 76 (1969), 169-171.

7. John Knopfmacher and Štefan Porubský, Topologies related to arithmetical properties of integral domains, Expos. Math. 15 (1997), 131-148.

8. František Marko and Štefan Porubský, A note on density and the Dirichlet condition, Int. J. Num. Theory (2012), 823-830.

9. Mangesh G. Murdeshwar, General topology, John Wiley \& Sons, Inc., New York, 1983.

10. Lynn Arthur Steen and J. Arthur Seebach, Jr., Counterexamples in topology, Dover Publications, Inc., Mineola, NY, 1995.

11. Paulina Szczuka, The connectedness of arithmetic progressions in Furstenberg's, Golomb's, and Kirch's topologies, Demonstr. Math. 43 (2010), 899-909.

12. , Connections between connected topological spaces on the set of positive integers, Cent. Europ. J. Math. 11 (2013), 876-881.

13. The Darboux property for polynomials in Golomb's and Kirch's topologies, Demonstr. Math. 46 (2013), 429-435. 
14. Lawrence C. Washington, Introduction to cyclotomic fields, Grad. Text. Math. 83, Springer-Verlag, New York, 1997.

Department of Mathematics, Oregon State University, Corvallis, Oregon 97331

Email address: orumj@oregonstate.edu 